\title{
Optical coherence tomography - a potential tool for roughness assessment of free and embedded surfaces of laser-machined alumina ceramic
}

\author{
R. Su, ${ }^{a}$ M. Kirillin, ${ }^{b}$ D. Jurków, ${ }^{c}$ K. Malecha, ${ }^{c}$ L. Golonka, ${ }^{c}$ L. Mattsson ${ }^{a}$ \\ ${ }^{a}$ Department of Production Engineering, KTH, the Royal Institute of Technology, Stockholm 10044, Sweden \\ ${ }^{b}$ Laboratory of Biophotonics, Institute of Applied Physics RAS, Nizhny Novgorod 603950, Russia \\ ${ }^{c}$ Faculty of Microsystems Electronics and Photonics, Wrocław University of Technology, Wrocław 50-372, Poland
}

\begin{abstract}
For an emerging market a mass and cost-effective production of non-silicon micro devices requires an inprocess and accurate 3D monitoring to assure the quality. Optical coherence tomography (OCT) providing highresolution contactless imaging is used to inspect the surface quality of a laser-machined alumina ceramic layer. Quantitatively the root mean square (rms) roughness is measured by traditional means and provides input parameters to the Monte Carlo simulation of OCT method. We found that the quality of both free and embedded surfaces of the highly-scattering alumina ceramic material can be evaluated by OCT.
\end{abstract}

Keywords: Alumina ceramics, optical coherence tomography (OCT), light scattering, surface roughness, Monte Carlo method, laser machining

\section{Introduction}

The technologies used in the manufacturing of silicon based microelectronic circuits have been widely spread to the micro manufacturing of complex 3D parts in other materials. In the emerging roll to roll manufacturing of ceramic micro devices advanced alumina materials have been considered to be important for several applications, such as Tera-Hertz components, cooler for automotive lighting systems, micro fluidic devices for micro reactors, fuel cells and medical devices. Some of the advantages are high thermal conductivity, hardness, low dielectric loss and stability against chemical attack. [1]

In the manufacturing process of these devices, which is based on a tape casting technique, laser machining is commonly used for the 3D-structuring of features in the tape, e.g. channels [2]. In order to get a good heat dissipation for from the surfaces of e.g. LED cooling systems, correct flow parameters are of primary importance. It is therefore important to ensure the feature dimensions, as well as the surface quality after a laser machining process.

However, it is very difficult to perform highresolution, in-process, and contactless inspection of the surface quality of machined micro-channels, particularly when the channels are embedded in a highly scattering ceramic material. Many mechanical and optical techniques [3] provide surface roughness assessment, but they have no access to embedded structures. Ultrasonic testing detects the internal microstructure and defects in materials [4], but has a significant drawback in process related characterization, as the test object has to be soaked in a liquid. X-ray micro tomography [5] is a very interesting technique with even sub-micron resolution, but the technique is by far too timeconsuming at present to be realistic as an inprocess metrology candidate.

Optical coherence tomography (OCT) on the other hand is considered to be one of the most successful non-destructive techniques providing depth profiles of internal and embedded structures.
Its non-invasive inspection technique combined with high resolution and relatively high data acquisition rate [6] has made it popular in the biomedical science. It has also been used for measuring surface roughness of milled metal, copper coins [7]. However, few have applied OCT for surface quality inspections of structured and/or embedded alumina ceramic layers. In this paper we have used a Talystep stylus profiler as a reference instrument for measuring surface roughness of $0.25 \mathrm{~mm}$ long profiles and the shape of profiles up to $1.6 \mathrm{~mm}$ long for alumina surfaces. We used the root mean square (rms) roughness values as input data for Monte Carlo simulations of the OCT response in the form of photon transport in highly scattering material. The results are shown as simulated OCT images [8] and they show the potential of using OCT for inspection of roughness of embedded surfaces.

\section{Experimental details \\ 2.1. Material}

The advantages mentioned above of alumina ceramic tapes for roll-to-roll micromanufacturing has resulted in a lot of attention. One important issue is to inspect surface quality of alumina layers and embedded channels or other structures. In the TeraHertz components for example surface roughness can affect the dielectric loss. For microfluidic devices the flow parameters of the liquid can be influenced by the surface quality.

The alumina tapes labelled MLA100 and SAL1 in our study were provided by Swerea IVF. The green tape contained $22 \%$ latex binder (LDM $7665 S$, Celanese). After sintering at $1630^{\circ} \mathrm{C}$ for 30 hours the binder was burnt away.

\subsection{Laser-machining}

Laser machining is one of the most popular methods for structuring ceramic green tapes [2]. The laser cutting is a very flexible non contact machining technique, which permits cutting materials at very high speeds (greater than 100 
$\mathrm{mm} / \mathrm{s}$ ) with high precision (beam diameter can be smaller than $20 \mu \mathrm{m}$ ). Therefore the method is very often applied in production and is commonly used in research labs. The cutting effect is achieved by vaporization of the machined material by absorption of the laser beam energy. The pulse energy depends on the average power and pulse repetition frequency of the laser while the pulse duration and beam wavelength affect the beam peak power and the energy absorptions of materials, respectively. The cutting speed and quality depends on machined material absorption coefficient of the laser wavelength. Therefore, different lasers and laser wavelengths are recommended for the machining of different materials. Laser cutting is commonly used in the fabrication of various multilayer ceramic sensors, actuators and microsystems.

\subsection{Optical coherence tomography}

The detailed principles of OCT can be found elsewhere [6]. Many varieties have been developed with various characteristics for different applications, towards the main objectives of higher resolution and imaging speed, and deeper penetration. A sweptsource Fourier-domain OCT (fig.1) is used in this study. At the exit of a Michelson interferometer the spatial frequency spectrum is recorded when tuning the light source and the power spectrum density is transferred to spatial information in axial direction according to the Wiener-Khinchin theorem. Only lateral scans are performed using galvo mirror, so the signal acquisition rate can be dramatically increased up to around $300 \mathrm{kHz}$. Moreover, the axial resolution determined by the coherence length of light source is decoupled with the lateral resolution that depends on the numerical aperture (NA) of objective. Thus structures embedded in translucent material or with high respect ratio can be detected without a severe aberration using a small NA.

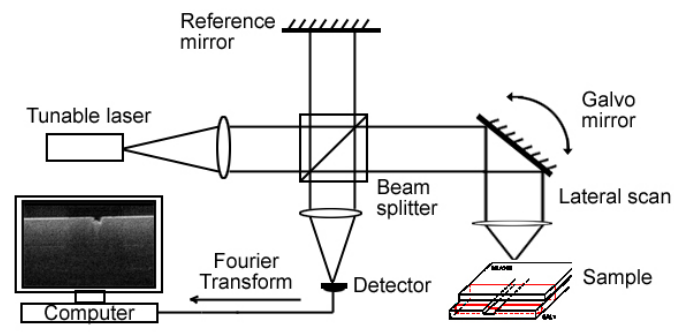

Fig.1. Schematic setup of OCT

\subsection{Monte Carlo simulation of OCT}

The basic idea of the Monte Carlo (MC) method is to generate a large number of "photons" with random properties and let them interact with the material through probability estimates of different scattering cross sections, absorption properties, refractive indices and angular dependencies. The results are obtained by statistical means. Applied to OCT in a scattering medium, the Monte Carlo method consists of numerous calculations of random photon trajectories based on the preset scattering medium characteristics.

For MC simulation of the OCT signal the distribution of the optical path lengths of the photon trajectories are crucial. Only the photons fulfilling the coherence gate and fitting the given detection conditions (detector position, size and numerical aperture) are selected [8]. These parameters were chosen based on the characteristics of experimental setup and optical properties of the studied material.

\subsection{Surface roughness}

Surface roughness is the measured quantity of the micro topography of a surface over a certain profile length (sampling length) and it has a strong correlation to the light scattering from the surface interface.[3] Many roughness parameters exist but In this paper we use the rms roughness $P q$ to represent the surface quality. It is equivalent to the standard deviation of the surface height variations and has a direct correspondence to the light scattering properties of smooth surfaces. The qualitative inspection of surface roughness obtained by the OCT measurements is further validated by the quantified measures from the Talystep which is used as a calibrated reference system.

\section{Results}

\subsection{Laser-machined channel}

The channel in SAL1 (see Fig.2) was cut (before sintering) with Nd-YAG laser (wavelength $1064 \mathrm{~nm}$, beam diameter $\sim 90 \mu \mathrm{m}$ ) at Thickfilm Microsystems Laboratory, Wrocław University of Technology. The variation of the laser beam diameter is around \pm 10 $\mu \mathrm{m}$, and the channel width is $\sim 160 \mu \mathrm{m}$ with a depth of $\sim 100 \mu \mathrm{m}$

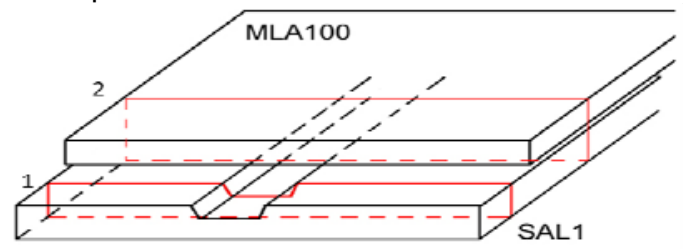

Fig.2. Geometrical layout of sample stack. Marked cross sections correspond to OCT imaging planes.

As shown in section 3.3 there is material built up (3-5 $\mu \mathrm{m})$ close to both sides of the channel and a considerably higher roughness has been measured in this area. The phenomena can be attributed to the strong heat transfer during laser cutting, which may change material properties of the machined tape and re-deposition of machined material. The material near the channel can probably be heated up to its sintering temperature. During sintering the preheated material (near the channel) may shrink in a different way than the bulk (far from channel). The slopes and higher roughness may also be created by deposition of vaporized material during the laser machining. The high laser energy is vaporizing the organic binder from the green tape and throws out ceramic grains that can be then accumulated near the channel, and create the slope and increased surface roughness on both sides of the channel. The phenomena are probably caused by both effects. Total understanding of the phenomena demands more investigations. 


\subsection{OCT images}

Michelson Diagnostics EX1301 MultiBeam OCT ( $\lambda 1.3 \mathrm{um}$ ) was used to evaluate the surface quality of the laser-machined alumina ceramics. The crosssection of the sintered SAL1 sample with its channel is illustrated in the OCT image of Fig. 3.

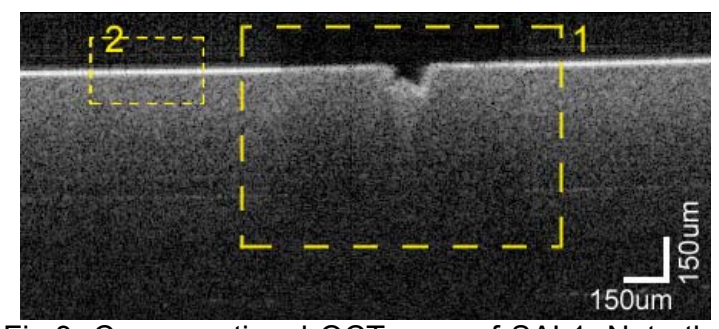

Fig.3. Cross-sectional OCT scan of SAL1. Note the brightness change at the interface in area 1 and 2 . The vertical bar presents the optical distance.

A darker region near the channel can be observed in Fig. 3. This is possibly caused by a) reduced backscattering within the material or by $b$ ) lowered penetration of the incident light. However, the tagged area in figure 4 suggests that the scattering coefficient in this region is not reduced as the probing depth is decreased. Alternative b) can be caused by either increased scattering at the upper interface or by increased absorption in the top part of the surface. The latter is negligible in sintered alumina at near infrared wavelength region.

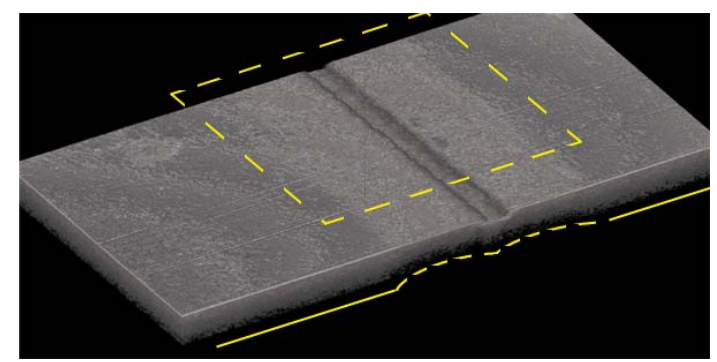

Fig.4. 3D representation of the multi slice OCT scans of the SAL1 (as same in fig.3); a typical scan of 400 slices would take about 40 seconds.

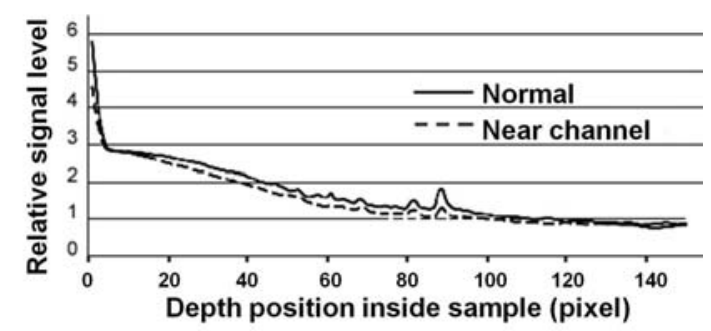

Fig.5. Mean OCT signal of A-scans near (dashed) and far from the machined channel (solid).

Fig. 5 shows the OCT response vs. depth close to and far from the channel. Near the channel (dashed line) the interface peak at depth 0 is lower and the intensity of scattered light is lower throughout the sampled depths. The main reason for this can be the higher roughness at the interface close to the channel. Increased scattering from a rough top surface would degrade the entrance peak, and scatter away the back reflected light returning from the deeper levels and result in the lowered light level in this region. The weakened peak at 90 pixels, corresponding to approximately $0.48 \mathrm{~mm}$ optical distance, proves again that the scattering within the material is not reduced. Otherwise the probing depth should be large enough to detect a pronounced peak. These consequences are also verified by the Talystep surface roughness measurement presented in the following section.

\subsection{Surface roughness measurement}

A $1.6 \mathrm{~mm}$ long primary profile of the sintered SAL1 surface is shown in Fig. 6. It was obtained with the KTH-Talystep surface profiler (tip radius 2 $\mu \mathrm{m})$ across the channel area. Surface roughness $P q$ (often referred to as rms roughness) was obtained from three $250 \mu \mathrm{m}$ long traces for each region marked I to IV in Fig. 6 . The rms roughness values and standard deviations are shown in Table1.

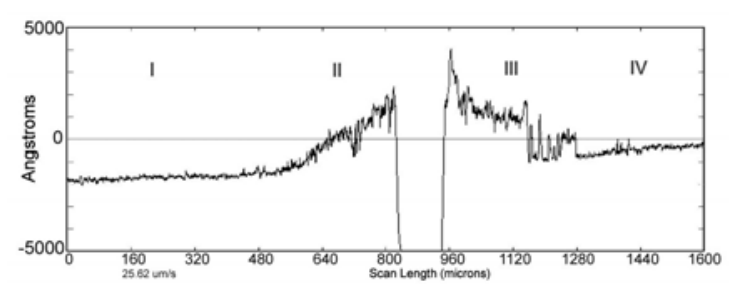

Fig.6. Primary surface profile perpendicular to the direction of the channel. Height scale $\pm 5 \mu \mathrm{m}$

Table1. Rms roughness $P q$ measured over $250 \mu \mathrm{m}$ in the four regions I - IV of Fig. 6.

\begin{tabular}{lcccc}
\hline Area & I & II & III & IV \\
\hline Ave $(n m)^{*}$ & 102 & 410 & 637 & 90 \\
\hline Std $(n m)^{* *}$ & 32 & 84 & 121 & 9 \\
\hline$\%$ STD & $32 \%$ & $21 \%$ & $19 \%$ & $10 \%$ \\
\hline
\end{tabular}

* Average;

** Standard deviation of three measurements.

As can be seen, areas II and III possess a surface roughness 4 to 6 times higher than the areas I and IV. And two slopes with a height of around $4 \mu \mathrm{m}$ over $300 \mu \mathrm{m}$ horizontal distance are observed near the channel. These findings are consistent with results of OCT measurement, and the phenomena caused during laser-cutting process as described in section 3.1.

\subsection{Monte Carlo simulations}

OCT simulations have been performed on the open surface of Fig. 3 as well as on the embedded structure of the alumina stack shown in Fig. 2. We will here only show the more challenging results of the stacked structure as the simulation results of the open structure is good agreement with the experimental results of Fig. 3. The performance of OCT on the stacked alumina structure was shown in a previous paper. [9] In the stacked case a $100 \mu \mathrm{m}$ sintered alumina top layer (MLA100) rests on the $300 \mu \mathrm{m}$ thick laser machined SAL1 sample with a $60 \mu \mathrm{m}$ air gap in between. In the simulation the channel depth was set to $90 \mu \mathrm{m}$, and the tapered channel had a width of $150 \mu \mathrm{m}$ at the top and $80 \mu \mathrm{m}$ at the bottom. The parameters used for the optical 
properties of the alumina are listed in table 2. The Heyney-Greenstein phase function is used to describe single scattering within the alumina.

Table2. Input parameters to the MC simulation

\begin{tabular}{ccc}
\hline $\begin{array}{c}\text { Scattering } \\
\text { Coefficient }\end{array}$ & $\begin{array}{c}\text { Anisotropy } \\
\text { Factor } \mathrm{g}\end{array}$ & $\begin{array}{c}\text { Axial and lateral } \\
\text { Resolution }\end{array}$ \\
\hline $20 \mathrm{~mm}^{-1}$ & 0.5 & $15 \mu \mathrm{m}$
\end{tabular}

${ }^{*}$ Average refractive index of our sintered alumina is 1.72 at operating wavelength $1.3 \mu \mathrm{m}$

The surface roughness data as shown in Fig. 6 and listed in Table 1 is only added to the left area of the simulated OCT image in Fig. 7. For comparison the right hand side of the channel has no surface roughness included in the simulation.

The MC simulation of the OCT response for the full alumina stack is shown in figure 7 . The optical path depth is given in $\mu \mathrm{m}$ on the Z-axis and the grayscale represents the signal strength. The channel bottom is clearly resolved under the 100 $\mu \mathrm{m}$ alumina layer confirming that the embedded channel is well assessed. The ideal sidewalls do not fulfill the geometry for backscattering and do not show up. By adding some surface roughness to them the backscattering can be increased.

Large differences in signal level and contrast can be found if comparing the left (rough interface) and the right hand side of the channel. At $x \sim 100$ $\mu \mathrm{m}$ the intensity level of the lower surface $(z \sim 490$ $\mu \mathrm{m})$ gradually decays, and almost disappears when approaching the channel. This tendency is clearly shown in area 1 of the measured OCT image in figure 3. As a comparison the simulated smooth surface on the right side in Fig. 7 shows a clearly detected boundary signal.

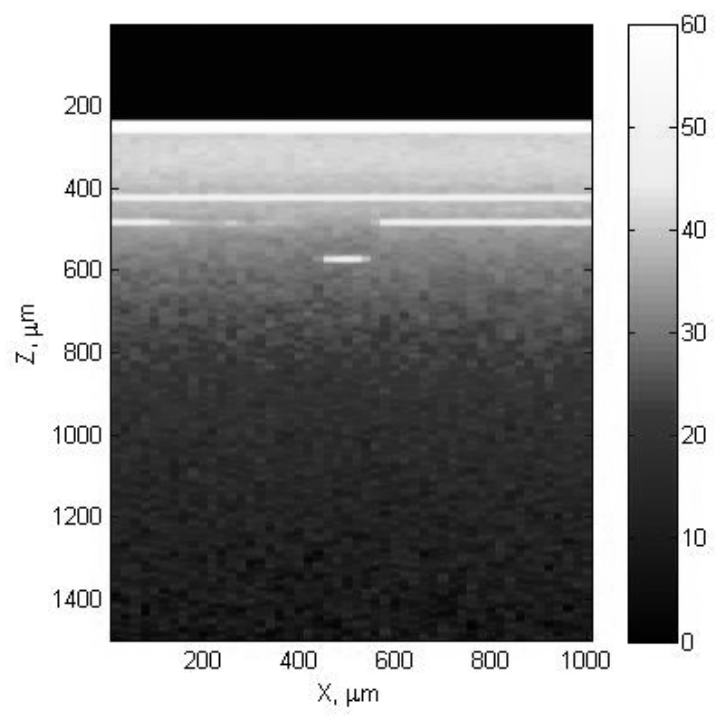

Fig.7. Simulated OCT image (in $\mathrm{dB}$ ) and optical depth $Z$ of the alumina stack vs $X$ position; lower interface include roughness to the left of the channel.

\section{Conclusion}

During laser-machining of alumina green tape preheating and vaporization can cause material to build up and add roughness to areas close to the processed area. In this paper we have investigated this effect on areas close to laser machined channels and showed that the roughened top or embedded surfaces can be detected as a reduced OCT signal. This was also verified by a Monte-Carlo simulation of the OCT signals with and without verified surface roughness values. The material build up and the roughness increase by a factor of 5 are considered as defects and can potentially cause quality problem in roll-to-roll manufacturing processes of microceramic devices, affecting the dielectric loss in Tera-Hertz components and the flow parameters of media in microfluidic devices.

\section{Acknowledgement}

The authors wish to thank Swerea IVF AB for providing the alumina samples and Michelson Diagnostics Ltd for the OCT images.

This work is carried out under the support of the FP-7 Multilayer project. It is partly supported by RFBR project 11-02-01129-a and FTP projects \# 02.740.11.0839 and 11.G34.31.0017. D. Jurków and K. Malecha acknowledge the Foundation for Polish Science (FNP) for financial support.

\section{References}

1. Overview. Retrieved from Multilayer: http://multilayer.4m-association.org/

2. J. Kita, A. Dziedzic, L. Golonka, T. Zawada (2002). Laser treatment of LTCC for 3D structures and elements fabrication. Microelectronics International, 19, 14-18.

3. J.M. Bennett, and L. Mattsson, (1999). Introduction to surface roughness and scattering (2nd ed.), Optical Society of America.

4. A.S. Birks, R.E. Green, Jr., technical editors; P. Mclntire, editor (1991). Ultrasonic testing, 2nd ed. American Society for Nondestructive Testing, ISBN 0931403049

5. S. R. Stock, (2008). MicroComputed Tomography: Methodology and Applications, CRC Press.

6. B. E. Bouma and G. J. Tearney, Handbook of optical coherence tomography, (Marcel Dekker, 2002).

7. T. Dresel, G. Häusler, H. Venzke, (1992). Threedimensional sensing of rough surfaces by coherence radar. Applied Optics , 31, 919-925.

8. M. Kirillin, I. Meglinski, V. Kuzmin, E. Sergeeva and R. Myllylä (2010). "Simulation of optical coherence tomography images by Monte Carlo modeling based on polarization vector approach", Optics Express, 18(21), 21714-21724

9. R. Su and L. Mattsson, "Depth profiling in alumina ceramic by optical coherence tomography," in Proc of the 4M 2010 Conference, eds. B Fillon, C. KhanMalek, S.Dimov, Bourg en Bresse November 17-19, 2010, pp 316-319 